\title{
Kinematics of deep inelastic scattering in leading order of the covariant approach
}

\author{
Petr Zavada* \\ Institute of Physics AS CR, Na Slovance 2, CZ-182 21 Prague 8, Czech Republic
}

\begin{abstract}
We study the kinematics of deep inelastic scattering corresponding to the rotationally symmetric distribution of quark momenta in the nucleon rest frame. It is shown that rotational symmetry together with Lorentz invariance can in leading order impose constraints on the quark intrinsic momenta. Obtained constraints are discussed and compared with the available experimental data.
\end{abstract}

PACS numbers: 12.39.-x 11.55.Hx 13.60.-r 13.88.+e

\section{INTRODUCTION}

The motion of quarks inside the nucleons plays an important role in some effects which are at present intensively investigated both experimentally and theoretically. Actual goal of this effort is to obtain a more consistent 3-D picture of the quark-gluon structure of nucleons. For example the quark transversal momentum creates the asymmetries in particle production in polarized (SIDIS) or in unpolarized (Cahn effect) experiments on deep inelastic scattering (DIS). Relevant tool for the study of these effects is the set of the transverse momentum dependent distributions (TMDs). Apparently, a better understanding of the quark intrinsic motion is also a necessary condition to clarify the role of quark orbital angular momenta in generating nucleon spin.

We have paid attention to these topics in our recent studies, see [1 5] and citations therein. In particular we have shown that the requirements of Lorentz invariance (LI) and the nucleon rotational symmetry in its rest frame $(\mathrm{RS})$, if applied in the framework of the 3$\mathrm{D}$ covariant quark-parton model (QPM), generate a set of relations between parton distribution functions. Recently we obtained within this approach relations between usual parton distribution functions and the TMDs. The Wanzura-Wilczek approximate relation (WW) and some other known relations between the $g_{1}$ and $g_{2}$ structure functions were similarly obtained in the same model before [7. Let us remark that the WW relation has been obtained independently also in another approaches [14, 15] in which the LI represents a basic input.

The aim of the present report is to consistently apply the assumption LI\&RS to the kinematics of DIS and to obtain the constraints on related kinematical variables. That is a complementary task to the study of above mentioned relations between distribution functions, which depend on these variables. So, the report can be considered as an addendum to our former papers related to the covariant QPM [1,9].

Since the present discussion is motivated and based on our earlier study of a covariant version of QPM, obtained results correspond only to the leading order of a

*Electronic address: zavada@fzu.cz more consistent QCD treatment. In this sense it would be interesting to compare our results with the leading order of a real QCD approach, like e.g. the recent study of perturbative QCD evolution of TMDs [11, 12. However such task would go beyond the scope of this short report. Anyway, in general a comparison between the experimental data and the leading order predictions can be important and instructive.

\section{KINEMATIC VARIABLES}

\subsection{The Bjorken variable and light-cone coordinates}

First, let us shortly remind the properties of the Bjorken variable

$$
x_{B}=\frac{Q^{2}}{2 P q},
$$

which plays a crucial role in phenomenology of lepton - nucleon scattering. Regardless of mechanism of the process, this invariant parameter satisfies

$$
0 \leq x_{B} \leq 1
$$

This is a very well-known textbook result. A possible proof is suggested also in [10]. Now let us consider a QPM approach, where the process of lepton - nucleon scattering is initiated by the lepton interaction with a quark (see Fig. 1), which obeys

$$
p^{\prime}=p+q, \quad p^{\prime 2}=p^{2}+2 p q-Q^{2} ; \quad Q^{2}=-q^{2} .
$$

The second equality implies

$$
Q^{2}=2 p q-\delta m^{2} ; \quad \delta m^{2}=p^{2}-p^{2},
$$

which with the use of relation (1) gives

$$
\frac{p q}{P q}=x_{B}\left(1+\frac{\delta m^{2}}{Q^{2}}\right) .
$$

The basic input for the construction of QPM is the assumption

$$
Q^{2} \gg \delta m^{2}
$$




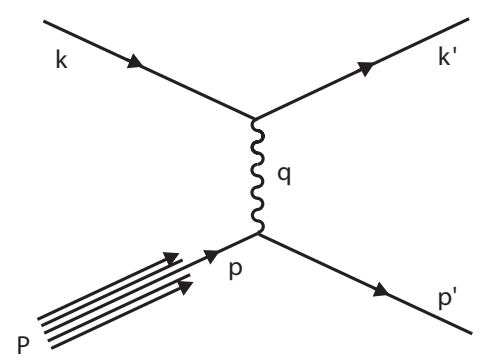

FIG. 1: Diagram describing DIS as a one photon exchange between the charged lepton and quark. Lepton and quark momenta are denoted by $k, p\left(k^{\prime}, p^{\prime}\right)$ in initial (final) state, $P$ is initial nucleon momentum.

which allows us to identify

$$
x_{B}=\frac{Q^{2}}{2 P q}=\frac{p q}{P q}
$$

and to directly relate the quark momentum to the parameters of scattered lepton. Moreover, if one assumes

$$
Q^{2} \gg 4 M^{2} x_{B}^{2}
$$

where $M$ is the nucleon mass, then one can identify

$$
x_{B}=x \equiv \frac{p_{0}-p_{1}}{P_{0}-P_{1}}
$$

in any reference frame in which the first axis orientation is defined by the vector $\mathbf{q}$. This relation can be proved as follows. Let us consider Eq. (7) in the same frame:

$$
x_{B}=\frac{p_{0} q^{0}-p_{1}|\mathbf{q}|}{P_{0} q^{0}-P_{1}|\mathbf{q}|} .
$$

In the nucleon rest frame we denote the photon momentum components by the subscript $R$ and using the usual symbol $\nu=q_{R}^{0}$ we have

$$
\left|\mathbf{q}_{R}\right|^{2}=\nu^{2}+Q^{2}
$$

which with the use of Eq. (1) gives

$$
\frac{\left|\mathbf{q}_{R}\right|^{2}}{\nu^{2}}=1+\frac{4 M^{2} x_{B}^{2}}{Q^{2}} .
$$

It means that for $Q^{2} \gg 4 M^{2} x_{B}^{2}$ we obtain

$$
\frac{\left|\mathbf{q}_{R}\right|}{\nu}=1+O\left(\frac{4 M^{2} x_{B}^{2}}{Q^{2}}\right) \text {. }
$$

(Since for $Q^{2} \rightarrow \infty$ we have also $\left|\mathbf{q}_{R}\right|, \nu \rightarrow \infty$, so the ratio $\left|\mathbf{q}_{R}\right| / \nu \rightarrow 1$ does not contradict Eq. (11).) In a reference frame connected with the rest frame by the Lorentz boost in the direction opposite to $\mathbf{q}_{R}$ we have the corresponding ratio

$$
\frac{q^{1}}{q^{0}}=\frac{\left|\mathbf{q}_{R}\right|+\beta \nu}{\nu+\beta\left|\mathbf{q}_{R}\right|}
$$

Now one can easily check that Eq. (7) with the use of this ratio and Eq. (13) imply

$$
x_{B}=\frac{p_{0} q^{0}-p_{1} q^{1}}{P_{0} q^{0}-P_{1} q^{1}}=\frac{p_{0}-p_{1}}{P_{0}-P_{1}}\left(1+O\left(\frac{4 M^{2} x_{B}^{2}}{Q^{2}}\right)\right) .
$$

In this way we have proved that replacement of Bjorken variable by the invariant light-cone ratio in Eq. (9) is valid provided the inequality $(8)$ is satisfied.

The relation (9) expressed in the nucleon rest frame reads

$$
x=\frac{p_{0}-p_{1}}{M},
$$

which after inserting into (2) gives

$$
0 \leq \frac{p_{0}-p_{1}}{M} \leq 1
$$

However the most important reason why we require large $Q^{2}$ is in physics. If we accept scenario when a probing photon interact with a quark, we need sufficiently large momentum transfer $Q^{2}$ at which the quarks can be considered as effectively free due to asymptotic freedom. At small $Q^{2}$ the picture of quarks (with their momenta and other quantum numbers) inside the nucleon disappear.

\subsection{Rotational symmetry}

The RS means that the probability distribution of the quark momenta $\mathbf{p}=\left(p_{1}, p_{2}, p_{3}\right)$ in the nucleon rest frame depends, apart from $Q^{2}$, on $|\mathbf{p}|$. It follows that also $-\mathbf{p}$ is allowed, so together with the inequality $(17)$ we have

$$
0 \leq \frac{p_{0}+p_{1}}{M} \leq 1
$$

The combinations of 17, , 18) imply

$$
0 \leq\left|p_{1}\right| \leq p_{0} \leq M, \quad\left|p_{1}\right| \leq \frac{M}{2} .
$$

And if we again refer to RS, then further inequalities are obtained:

$$
0 \leq|\mathbf{p}| \leq p_{0} \leq M, \quad|\mathbf{p}| \leq \frac{M}{2}, \quad 0 \leq p_{T} \leq p_{0} \leq M
$$

and

$$
p_{T} \leq \frac{M}{2},
$$

where

$$
|\mathbf{p}|=\sqrt{p_{1}^{2}+p_{2}^{2}+p_{3}^{2}}, \quad p_{T}=\sqrt{p_{2}^{2}+p_{3}^{2}} .
$$

Obviously inequality (21) is satisfied also in any reference frame boosted in the directions $\pm \mathbf{q}$. Further, the above 
inequalities are apparently valid also for average values $\left\langle p_{0}\right\rangle,\left\langle p_{1}\right\rangle,\langle|\mathbf{p}|\rangle$ and $\left\langle p_{T}\right\rangle$. In addition, if one assumes that $p_{T}-$ distribution is a decreasing function, then necessarily

$$
\left\langle p_{T}\right\rangle \leq \frac{M}{4} .
$$

The above relations are valid for sufficiently high $Q^{2}$ suggested by Eqs. (6) and (8). Let us note that the on-mass-shell assumption has not been applied for obtaining these relations.

These inequalities can be compared with relations obtained in [9, where the additional on-mass-shell condition $m^{2}=p^{2}=p_{0}^{2}-\mathbf{p}^{2}$ had been applied. Corresponding relations are more strict:

$$
\frac{m^{2}}{M^{2}} \leq x \leq 1, \quad p_{0} \leq \frac{M^{2}+m^{2}}{2 M}, \quad|\mathbf{p}| \leq \frac{M^{2}-m^{2}}{2 M}
$$

and

$$
p_{T}^{2} \leq M^{2}\left(x-\frac{m^{2}}{M^{2}}\right)(1-x) .
$$

However, it is clear that in general the on-mass-shell assumption is not realistic. In the next we will assume only the off-mass-shell approach.

\section{DISCUSSION}

First let us summarize more accurately what we have done in the previous section. We assumed:

a) Lorentz invariance

It means that the theoretical description in terms of the standard kinematical variables (see Fig. 1)

$$
q, x_{B}, x, p=\left(p_{0}, p_{1}, p_{2}, p_{3}\right), P=\left(P_{0}, P_{1}, P_{2}, P_{3}\right)
$$

can be boosted also to the nucleon rest frame.

b) Inequality $0 \leq x \leq 1$

It means that the light-cone ratio $x$ satisfies the same bound (2) as the Bjorken variable $x_{B}$.

c) Rotational symmetry

The kinematical region $\mathcal{R}^{3}$ of the quark intrinsic momenta $\mathbf{p}=\left(p_{1}, p_{2}, p_{3}\right)$ in the nucleon rest frame has rotational symmetry (i.e. $\mathbf{p} \in \mathcal{R}^{3} \Rightarrow \mathbf{p}^{\prime}=\mathbf{R} \mathbf{p} \in \mathcal{R}^{3}$, where $\mathbf{R}$ is any rotation in $\mathcal{R}^{3}$ ). For example, in terms of the covariant QPM it means that probabilistic distribution of the quark momenta is controlled by some function $G\left(p P / M, Q^{2}\right)$.

We proved these assumptions imply bounds 17$)-(21)$. Now we will shortly comment on the obtained results:

i) The ratio $x$ of light-cone variables $(9)$ has a simple interpretation in a frame, where the proton momentum is large $-x$ is the fraction of this momentum carried by the quark. However an interpretation of the same variable in the nucleon rest frame is more complicated. In this frame the quark transversal momentum cannot be neglected and $x$ depends on the both, longitudinal and transversal quark momenta components. In the limit of massless quarks the connection between the variable $x$ in (16) and the quark momenta components is given by the relations:

$$
\begin{aligned}
x & =\frac{p_{0}-p_{1}}{M} ; & p_{0} & =\sqrt{p_{1}^{2}+p_{T}^{2}}, \\
p_{1} & =-\frac{M x}{2}\left(1-\frac{p_{T}^{2}}{M^{2} x^{2}}\right), & p_{0} & =\frac{M x}{2}\left(1+\frac{p_{T}^{2}}{M^{2} x^{2}}\right) .
\end{aligned}
$$

These variables were used in our recent papers on TMDs [1, 2. Simply the value of invariant variable $x$ does not depend on the reference frame, but its interpretation e.g. in the rest frame differs from that in the infinite momentum frame.

ii) The relations 201, 21), which follow from RS, can be confronted with the experimental data on $\left\langle p_{T}\right\rangle$ or $\langle|\mathbf{p}|\rangle$. We have discussed the available data in [1, 2] and apparently relation (21) is compatible with the set of lower values $\left\langle p_{T}\right\rangle$ corresponding to the 'leptonic data'. On the other hand the second set giving substantially greater $\left\langle p_{T}\right\rangle$ and denoted as the 'hadronic data', seems to contradict this relation. Actually the conflict with the relation (21) would mean either the conflict with some of the assumptions $a$ ) $-c$ ) or simply it can be due to absence of the higher order QCD corrections. However, for possible comparison with the perturbative QCD approach [11] let us remark the following. This approach generate evolved TMDs using as input the existing phenomenological parametrizations extracted from the experimental data. For example, one of the inputs is the scale-independent Gaussian fit [13]

$$
F_{f / P}\left(x, p_{T}\right)=f_{f / P}(x) \frac{\exp \left[-p_{T}^{2} /\left\langle p_{T}^{2}\right\rangle\right]}{\pi\left\langle p_{T}^{2}\right\rangle},
$$

where $\left\langle p_{T}^{2}\right\rangle=(0.38 \pm 0.06) \mathrm{GeV}^{2}$. Obviously our concept RS defined above is hardly compatible with this distribution. In fact in the rest frame this distribution gives much greater $\left\langle p_{T}^{2}\right\rangle$ than the corresponding longitudinal term $\left\langle p_{1}^{2}\right\rangle$. However RS requires $\left\langle p_{T}^{2}\right\rangle=2\left\langle p_{1}^{2}\right\rangle$ only. Let us remark that this imbalance is of the same order as a difference between the two data sets mentioned above.

iii) The first relation (20) apparently contradicts an intuitive, Lorentz invariant condition

$$
|\mathbf{p}|^{2}>p_{0}^{2}
$$

corresponding to bound, space-like quarks. Such conflict does not take place for the leading order with quarks onmass-shell. However, for off-shell quarks the condition (27) is incompatible with the assumptions $a$ ) $-c$ ), which imply (20). For example it means that any approach, which is based primarily on the assumption (27) in some starting reference frame (including the infinite momentum frame) cannot simultaneously satisfy the conditions a) $-c)$.

iv) In 2 we explained why the RS, if applied on the level of QPM, follows from the covariant description. In 


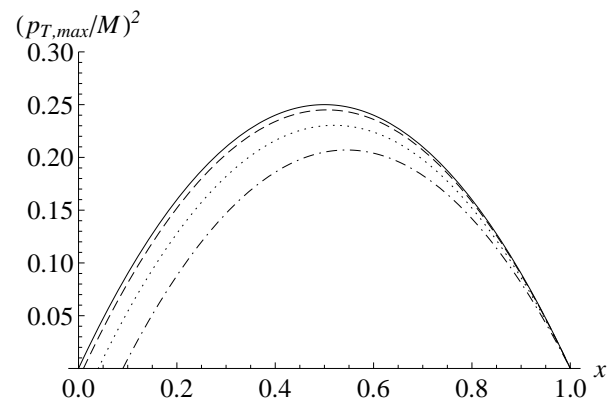

FIG. 2: Upper limit of the quark transversal momentum as a function of $x$ for $\mu=0$ (solid line), 0.1 (dashed line), 0.2 (dotted line) and 0.3 (dash-dotted line).

fact it means the assumptions $a$ ) $-c$ ) are common for our QPM and for the approaches like [14, 15] where only Lorentz invariance is explicitly required. The predictions of all these models are compatible with the bound (21). This is just a consequence of the fact that general conditions $a$ ) -c) are satisfied in these approaches. Another theoretical reasons for RS have been suggested in [8]. Let us remark that the rotational-symmetry properties of the nucleon state in its rest frame play an important role also in the recent studies [17].

v) The relation (24) is obtained for the quarks on-massshell. In a more general case, where only inequalities (20), (21) hold, this relation is replaced by

$$
p_{T}^{2} \leq M^{2}\left(x-\frac{\mu^{2}}{M^{2}}\right)(1-x) ; \quad \mu^{2} \equiv p_{0}^{2}-\mathbf{p}^{2},
$$

where the term $\mu^{2}$ is not a parameter corresponding to the fixed mass, but it is only a number varying in the limits defined by (20). The last relation implies for any $\mu^{2}$ :

$$
p_{T}^{2} \leq M^{2} x(1-x)
$$

which is equivalent to the on-mass-shell relation 24 for $m=0$. This general upper limit for $p_{T}^{2}$ depending on $x$ is displayed in Fig. 2. Let us remark that results on $\left\langle p_{T}^{2}(x)\right\rangle$ obtained in [14, 15] are compatible also with the bound (29). An equivalent form of this inequality was probably for the first time presented in [18.

\section{SUMMARY}

In the present report we studied the kinematic constraints due to the rotational symmetry of the quark momenta distribution inside the nucleon in the leading order approach. In particular, we have shown that the light-cone formalism combined with the assumption on the rotational symmetry in the nucleon rest frame imply $p_{T} \leq M / 2$. Only part of existing experimental data on $\left\langle p_{T}\right\rangle$ satisfies this bound, but the another part does not. In general, the existing methods for reconstruction of $\left\langle p_{T}\right\rangle$ from the DIS data are rather model-dependent. These are the reasons why more studies are needed to clarify these inconsistencies, since the phenomenological distributions in the $x-p_{T}$ plane at present serve also as an input for a more fundamental calculation of the QCD evolution and other effects.

\section{Acknowledgments}

This work was supported by the project AV0Z10100502 of the Academy of Sciences of the Czech Republic. I am grateful to Anatoli Efremov, Peter Schweitzer and Oleg Teryaev for many useful discussions and valuable comments.
[1] A. V. Efremov, P. Schweitzer, O. V. Teryaev and P. Zavada, Phys. Rev. D 83, 054025 (2011).

[2] P. Zavada, Phys. Rev. D 83, 014022 (2011) arXiv:0908.2316 [hep-ph]].

[3] A. V. Efremov, P. Schweitzer, O. V. Teryaev and P. Zavada, PoS DIS2010, 253 (2010) arXiv:1008.3827 [hep-ph]].

[4] A. V. Efremov, P. Schweitzer, O. V. Teryaev and P. Zavada, Phys. Rev. D 80, 014021 (2009).

[5] P. Zavada, Eur. Phys. J. C 52, 121 (2007).

[6] A. V. Efremov, O. V. Teryaev and P. Zavada, Phys. Rev. D 70, 054018 (2004).

[7] P. Zavada, Phys. Rev. D 67, 014019 (2003).

[8] P. Zavada, Phys. Rev. D 65, 054040 (2002).

[9] P. Zavada, Phys. Rev. D 55, 4290 (1997).

[10] P. Zavada, arXiv:1106.5607 [hep-ph] v1.
[11] S. M. Aybat and T. C. Rogers, Phys. Rev. D 83, 114042 (2011) arXiv:1101.5057 [hep-ph]].

[12] S. M. Aybat, A. Prokudin and T. C. Rogers, arXiv:1112.4423 [hep-ph].

[13] P. Schweitzer, T. Teckentrup and A. Metz, Phys. Rev. D 81, 094019 (2010) arXiv:1003.2190 [hep-ph]].

[14] U. D'Alesio, E. Leader and F. Murgia, Phys. Rev. D 81, 036010 (2010) arXiv:0909.5650 [hep-ph]].

[15] J. D. Jackson, G. G. Ross and R. G. Roberts, Phys. Lett. B 226, 159 (1989).

[16] R. K. Ellis, W. Furmanski and R. Petronzio, Nucl. Phys. B 212 (1983) 29; Nucl. Phys. B 207, 1 (1982).

[17] C. Lorce' and B. Pasquini, arXiv:1111.6069 [hep-ph], arXiv:1109.5864 [hep-ph], arXiv:1107.3809 [hep-ph].

[18] J. Sheiman, Nucl. Phys. B171, 445 (1980). 